\title{
Exploring the relationship between pain intensity and knee moments in participants with medial knee osteoarthritis: a cross- sectional study
}

Chen. Huang ${ }^{1}$, Ping-Keung. Chan², Kwong-Yuen. Chiư ${ }^{2}$ Chun-Hoi. Yan², Shun-Shing. Yeung ${ }^{3}$ and Siu N. Fu ${ }^{1 *}$

\begin{abstract}
Background: High biomechanical loading is believed to be a risk factor to pain in people with knee osteoarthritis $(\mathrm{OA})$, but controversial findings have been reported on the relationship between external knee adduction moment (KAM) and pain. A more comprehensive analysis considering other factor such as external knee flexion moment (KFM) could help better reveal this relationship. This study explored the relationship between external knee adduction moment and pain intensity in participants with knee osteoarthritis $(\mathrm{OA})$ using an integrated path analysis model.

Methods: This was a cross-sectional study based on laboratory setting. Forty-seven participants with clinical and radiographic medial knee OA were analyzed for their external knee adduction moment (KAM) and knee flexion moment (KFM) during walking using a motion analysis system. Pain intensity was measured by visual analogue scale (VAS) and the pain subscale of the Knee Injury and Osteoarthritis Outcome Score. Varus/valgus alignment was captured and quantified using a bi-planar X-ray system. Using a path analysis model, the relationships between pain intensity, KAM, KFM, OA radiographic severity, knee varus angle and walking speed were examined.

Results: The proposed path model met the goodness-of-fit criteria. Based on this model, KAM had a negative effect on VAS pain indirectly through the mediation of KFM. The model indicated KAM and KFM were negatively related to one another; and KFM was positively related to VAS. The KAM index, defined as (KAM/ (KAM + KFM)), was negatively related to VAS.

Conclusions: Path analysis enabled the construction of a more integrated pathokinematic framework for people with knee $\mathrm{OA}$. The KAM index which reflected the load sharing on the frontal and sagittal planes also revealed its relationship with pain. Re-distribution of mechanical loading from frontal to sagittal plane might be a strategy for pain avoidance associated with mechanical irritation.
\end{abstract}

Keywords: Knee osteoarthritis, External knee adduction moment, Pain

\footnotetext{
* Correspondence: amy.fu@polyu.edu.hk

'Department of Rehabilitation Sciences, The Hong Kong Polytechnic University, Hung Hom, Hong Kong, China

Full list of author information is available at the end of the article
}

(C) The Author(s). 2021 Open Access This article is licensed under a Creative Commons Attribution 4.0 International License, which permits use, sharing, adaptation, distribution and reproduction in any medium or format, as long as you give appropriate credit to the original author(s) and the source, provide a link to the Creative Commons licence, and indicate if changes were made. The images or other third party material in this article are included in the article's Creative Commons licence, unless indicated otherwise in a credit line to the material. If material is not included in the article's Creative Commons licence and your intended use is not permitted by statutory regulation or exceeds the permitted use, you will need to obtain permission directly from the copyright holder. To view a copy of this licence, visit http://creativecommons.org/licenses/by/4.0/ The Creative Commons Public Domain Dedication waiver (http://creativecommons.org/publicdomain/zero/1.0/) applies to the data made available in this article, unless otherwise stated in a credit line to the data. 


\section{Background}

Knee osteoarthritis (OA) is a common problem in the senior population worldwide [1] and activity-related pain is the most predominant disabling symptom of this condition [2]. More importantly, there is an upward trend in the prevalence of knee pain associated with OA [3]. In people over 55 years of age, about $10 \%$ had mild-to-moderate knee pain, and between 2.0 and $4.8 \%$ would suffer from severe pain and disability caused by OA [4, 5]. The pain and disability would lessen their willingness to participate in physical and social activities [6], and strongly affect their health-related quality of life [7].

Most pain in knee OA is activity triggered especially in weight-bearing situations [8,9]. Extrinsic and intrinsic factors that increase joint mechanical loading lead to greater intensity of knee pain [10]. Physically demanding occupations, habitual and intense physical activities had strong relationship with knee pain and joint degeneration [11]; people who were over-weight had over two fold higher risk of knee OA and obesity was related to $24.6 \%$ of new onset of knee pain [11]. Excessive knee loading during gait, in particular, the external knee adduction moment (KAM), has been proposed as an essential intrinsic factor for OA related pain. Amin et al. found that seniors with higher peak KAM were more likely to develop chronic knee pain within 3-4 years [12]. Nevertheless, studies in search of relationship between KAM and pain intensity reported inconsistent findings [13-16]. In participants with mild radiographic knee OA, peak KAM was significantly higher in the symptomatic than the asymptomatic groups $[13,16]$. However, a negative relationship between peak KAM and pain intensity was reported by Henriksen et al. [15]. It was also found that greater KAM impulse was related to higher pain intensity in participants with moderate radiographic knee OA $[14,15]$, but this was associated with lower pain intensity in participants with severe radiographic knee OA [14]. It is essential to consider the degree of OA severity when exploring the relationship between KAM and pain intensity in view of the fact that such a relationship was very likely to be specific to radiographic severity,

Peak external knee flexion moment (KFM) reflected joint loading, and the load would trigger pain in participants with knee OA. In people with symptomatic mild knee OA, the KFM at early stance phase was lower than their asymptomatic counterparts [13], but people with higher KFM were more likely to develop pain after exercises [17]. In view that KAM and KFM occur nearly simultaneously with the first peak of medial joint contact force at about the initial $23 \%$ of the total gait cycle [18], Simic et al. reported that increase in KFM was associated with a reduction in KAM with gait modification
[19]. They also found that KAM would drop but KFM would rise with toe-in gait; whereas the opposite was observed with toe-out gait during the first half of stance phase in people with knee OA [19]. These findings suggested an inverse relationship existed between KAM and KFM [20]. In order to better understand the relationship between KAM and pain, an analysis on the simultaneous change between KAM and KFM when taking into considerations of factors such as joint alignment [21] and walking speed [22] might better explain the direct relationship between KAM and pain.

Load sharing among the three anatomical planes has emerged as one of the mechanical outcome considerations in participants with knee OA. Asay et al. reported a transition of KFM-dominated total joint loading to a KAM-dominated loading in the long term, and the percentage of KAM over the total joint moment appeared to be associated with radiographic OA progression at follow-ups over a period of 5 years [23]. The percentage of KAM in total joint moment was associated with the change in medial-to-lateral knee articular cartilage thickness ratio in an 8-year follow-up. Hence, the proportion of KAM was possibly one key biomechanical factor linking to joint structure destruction in the initiation and progression of knee OA. Besides, the KFM and KAM contributed to $73 \%$ of variance of the total joint force [24]. The external knee moment on the horizontal plane accounted for less than $1 \%$ of total external joint moment [23] which was relatively low during the stance phase, thus its influence had been less emphasized. Therefore we aimed to explore whether the KAM index, which was the percentage of KAM over the sum of KAM and KFM, was associated with pain intensity in participants with medial knee OA. A cross-sectional relationship between KAM index and OA-related pain would help to establish their causal relationship.

The main goal of this study was to investigate the relationships between KAM and pain intensity in people with mild-to-moderate medial knee OA by path analysis taking the effects of KFM, disease severity, joint alignment and walking speed into considerations. We also explored whether load sharing represented by KAM index would have a relationship with pain intensity. It was hypothesized that (1) pain intensity could be determined by early stance KAM directly and indirectly through KFM; (2) there would be a positive association between KAM index and pain intensity in participants with mild-to-moderate medial knee OA.

\section{Methods \\ Study design}

This was an observational cross-sectional study. 


\section{Participants}

Participants were recruited from orthopedic department of a local hospital. The inclusion criteria were: (1) age between 50 and 80 years; (2) had minimal knee pain of 2 on an 11-point visual analogy scale (VAS) during level walking and with most painful site located in the medial knee compartment; and (3) plain x-ray revealed more degenerative changes in the medial than lateral compartment. Participants were excluded if they had any of the following: (1) history of low back or lower limb injury within the past 12 months; (2) low back, pelvis, hip, ankle or foot pain of VAS 3 or above; (3) rheumatoid arthritis, (4) knee valgus more than $3^{\circ}$ [25], (5) history of knee surgery; (6) history of intra-articular injection in the past 3 months; (7) any other muscular, joint or neurological condition influencing lower limb function; (8) unable to walk independently without external assistance; or (9) body mass index (BMI) $>36 \mathrm{~kg} / \mathrm{m}^{2}$ [14]. Study size was reached according to the availability of eligible participants at the study period. Mild knee OA was identified as grade 1 and 2 in Kellgren-Lawrence (KL) grading scale and moderate groups as grade 3 [15].

\section{Outcome measurement \\ Pain intensity}

Pain intensity was measured by both VAS and the pain subscale of the Knee Injury and Osteoarthritis Outcome Score (KOOS). The maximal level of pain intensity during walking in the past week was measured by an 11point VAS with "0" represented no pain and 10 represented the worst pain. It quantified pain intensity in particular during walking in particular. Pain subscale from KOOS was also used to assess the pain intensity more comprehensively in different functional conditions instead of just walking as measured with the VAS. For each subscale, a 0-100 score scale was used with "0" represented the most severe knee problem, while " 100 " indicated no problem; thus higher scores indicated less pain. Convincing evidence from meta-analysis suggested KOOS had adequate content validity, internal consistency and construct validity [26], and excellent test-retest reliability of translated version has also been reported [26]. Participants were required to complete the subscale without any assistance from the assessor.

\section{Knee joint kinetics}

Knee joint kinetics was measured by a motion analysis system comprising 8 cameras (MX T40, Vicon, Oxford, UK) and 2 floor-mounted force plates (Kistler Group, Winterthur, Switzerland). The frame rate for the kinetic data was $100 \mathrm{~Hz}$. Lower limb anthropometric information including knee width, ankle width and lower limb length were recorded. Sixteen reflective skin markers were attached according to the standard lower limb
Plug-In-Gait marker set. The skin markers included bilateral anterior superior iliac spine, posterior superior iliac spine, thigh, knee, tibia, ankle, toe and heel [14]. Participant stood unshod and an initial recording was made during standing for lower limb modeling purpose. Afterwards, participants walked unshod in their comfortable speed on an 8-meter footpath. Adequate practice trials were provided for the participants to acquaint themselves with the test. Each participant was recorded for at least five walking trials. The five trials would be accepted for analysis only if both lower limbs had clean foot strike from heel-strike to toe-off on the force plates [27].

Vicon Nexus software (Version 2.5, Oxford, UK) was used to estimate the external knee joint moments with Lower limb Plug-In-Gait model settings. The periods between toe-off and heel strike were identified as when the magnitude of the force plate was below $10 \mathrm{~N}$. Half of the stance phase was marked and peak KAM and KFM were respectively defined as the maximum values of knee moment in the frontal and sagittal plane during the initial $50 \%$ of stance phase. They were normalized to body weight and reported in $\mathrm{Nm} / \mathrm{kg}$. All kinetic data were estimated by the average of five successful trials. KAM index was calculated as (KAM/ KAM + KFM)*100 as derived from Asay et al. [28].

\section{Knee varus/valgus angle}

Knee varus/valgus angle was examined with a low-dose bi-planar X-ray imaging system (EOS imaging, Paris, France) [29]. Participants stood with legs $4 \mathrm{~cm}$ apart so as to obtain a clear image of both legs. Knee varus/valgus was measured as the angle between the longitudinal axes of femur and tibia via the sterEOS software. (Version 1.6, EOS imaging, Paris, France). Anatomical reference points were identified on both the sagittal and coronal planes, and bony contours were adjusted according to the EOS guidelines. Between-day reliability was assessed in six participants and the result was excellent $(\mathrm{ICC}=0.99, p<0.001)$.

\section{Statistical analysis}

Statistical analysis was conducted with $\mathrm{R}$ software (Version 4.02). For participants with bilateral symptoms, analyses were conducted on the more painful leg. All the variables were normally distributed as assessed by Shapiro-Wilk test. Correlations between VAS pain, KOOS pain, KAM, KFM, radiographic severity, knee varus angle and walking speed were assessed by twotailed Pearson correlation coefficient. The relationship between pain intensity and KAM were estimated by path analysis with maximum likelihood estimation. There was one missing data in the KOOS pain subscale and it was handled by pairwise deletion in the analysis. 
Path analysis was an extension of multiple regression analysis using correlational data to discover the strength of effect of a hypothesized system [30]. It had the advantage to estimate both direct and indirect effects between variables. Pain intensity, KAM and KFM were the endogenous variables whereas walking speed, OA radiographic severity and knee varus angle were the exogenous variables. The results of correlation between exogenous variables were not shown in the model. The goodness-of-fit criteria was assessed by Chi-square, Comparative Fit Index, Tucker-Lewis Index, Root Mean Square Error of Approximation and Standardized Root Mean Square Residual [31]. The model proposed in this study was a conceptual model of the relationships between knee joint loadings and pain intensity sourced from the literature.

Correlations between VAS pain, KOOS pain and KAM index were assessed by partial Pearson correlation coefficient test controlling for radiographic severity, knee varus angle and walking speed.

\section{Results}

A total of 100 participants with knee OA were initially screened and 47 who satisfied the study criteria were recruited. Main reasons for exclusion were low back pain, recent knee injury or surgery and lateral knee OA. The Information of the included participants was shown in Table 1. Their mean age was $62.1 \pm 6.0$ years old and $78 \%$ of the participants were females. Thirty participants (64\%) were categorized as mild knee OA. All except 4 participants had bilateral knee OA.

Table 1 Descriptive information

\begin{tabular}{ll}
\hline Characteristics & $(\boldsymbol{n}=\mathbf{4 7})$ \\
\hline Demographic information & \\
Age (years) & $62.06 \pm 6.01$ \\
BMI $\left(\mathrm{kg} / \mathrm{m}^{2}\right)$ & $26.25 \pm 3.56$ \\
Gender (female/male) & $37 / 10$ \\
Mild/Moderate & $30 / 17$ \\
Bilateral/Unilateral & $43 / 4$ \\
Knee varus angle $\left(^{\circ}\right)$ & $6.02 \pm 5.27$ \\
Walking speed (m/s) & $1.00 \pm 0.17$ \\
Knee joint kinetics & \\
External knee adduction moment $(\mathrm{Nm} / \mathrm{kg})$ & $0.50 \pm 0.15$ \\
External knee flexion moment $(\mathrm{Nm} / \mathrm{kg})$ & $0.47 \pm 0.26$ \\
KAM index (\%) & $54.12 \pm 17.41$ \\
Pain & \\
VAS & $5.15 \pm 1.76$ \\
KOOS & $63.08 \pm 14.02$ \\
\hline
\end{tabular}

\section{Relationships between self-perceived pain and joint loading}

As shown in Table 2, VAS self-perceived pain intensity was positively related to KFM $(r=0.43, p=0.003)$ and it had a negative association with KAM $(r=-0.29, p=0.05)$. The KAM had a negative relationship with KFM $(\rho=-$ $0.40, p=0.01$ ) and positive association with knee varus angle $(r=0.55, p<0.001)$. There was less likely any relationship between KOOS pain intensity and KAM $(r=-0.13, p=0.40)$ or KFM $(r=-0.01, p=0.63)$. Nevertheless, greater pain intensity measured by VAS was associated with greater pain intensity measured by the $\operatorname{KOOS}(r=-0.39, p=0.01)$.

\section{Path analysis}

The model including VAS pain intensity was examined to be of good fit according to Chi-square test $\left(\chi^{2}=4.89\right.$, $\mathrm{df}=4, p=0.30)$, Comparative Fit Index $(\mathrm{CFI}=0.98)$ and Tucker Lewis Index (TLI $=0.93)$, which met the cut-off criterion [32]. The value for Standardized Root Mean Square Residual was 0.06 and that for Root Mean Square Error of Approximation was 0.07, and both were smaller than the threshold of 0.08 . The effects of path model were shown in Table 3.

KAM was found to have an indirect effect on VAS pain intensity through KFM (see Table 4). No direct effect was found between KAM and VAS pain intensity. However, there existed a moderate negative relationship between KAM and KFM; the magnitude of KFM had a direct effect on VAS pain intensity. The effect of radiographic severity, knee varus angle and walking speed had been demonstrated in the path model (Table 3; Fig. 1).

Relationships between self-perceived pain and KAM index Figure 2 showed the scatter plot between KAM index and pain intensity. There existed a negative association between the two variables $(r=-0.45, p=0.002)$ after controlling for radiographic severity, knee varus angle and walking speed. Hence, higher intensity of pain was associated with lower KAM index in participants with knee OA.

\section{Discussion}

This study aimed to explore the relationships between pain intensity and knee joint loading in people with mild-to-moderate medial knee OA. Using path analysis, a more integrated conceptual framework was constructed. The findings suggested KAM had a negative effect on pain with KFM as its mediator. By using the KAM index, we also revealed a negative relationship with pain intensity in our participants.

Based on these findings, it is suggested that magnitude of KAM had a negative effect on pain intensity. The effect of KAM on pain was through the mediation of 
Table 2 Correlation coefficient of variables in path analysis

\begin{tabular}{|c|c|c|c|c|c|c|c|}
\hline & VAS Pain & KOOS pain & KAM & KFM & Severity & Walking speed & Knee varus angle \\
\hline VAS pain ${ }^{a}$ & & $-0.39 * *$ & $-0.29^{*}$ & $0.43^{* *}$ & 0.27 & -0.02 & 0.04 \\
\hline KOOS pain ${ }^{a}$ & & & -0.13 & -0.07 & 0.01 & 0.01 & -0.05 \\
\hline KAM & & & & $-0.40^{* *}$ & 0.18 & 0.01 & $0.55^{* *}$ \\
\hline KFM & & & & & 0.01 & 0.15 & -0.06 \\
\hline Severity & & & & & & $-0.33^{*}$ & 0.62 \\
\hline Walking speed & & & & & & & $-0.31^{*}$ \\
\hline
\end{tabular}

* $p<0.05,{ }^{* *} p<0.01$

${ }^{a}$ High VAS pain score indicates high pain intensity; low KOOS pain score indicates high pain intensity

KFM. Negative relationship between VAS pain and KAM were reported in previous studies in people with mild radiographic severity of OA $[15,33]$. The authors proposed such relationship could be an avoidance mechanism in response to pain provoking stimuli. Indeed, experimental pain induced in infrapatellar fat pad would significantly reduce the KAM and KFM during gait in healthy participants [34]. The authors further proposed the modulation on KAM and KFM was related to alteration in trunk motion, foot progression angle or muscle-coordination. However,
OA-related pain is usually chronic and trigged by loading activity [9] and findings from healthy participants with experimentally induced pain might not be translated to participants with knee OA. Besides, during gait modification, reduction in KAM was associated with increase in KFM in participants with knee OA [34] which agreed with the present finding that moderate negative association was detected between KAM and KFM. In participants with knee OA, mechanical modification was proposed as the mechanism for the interchange between KAM and KFM.

Table 3 Decomposition of effects from path analysis

\begin{tabular}{|c|c|c|c|c|c|}
\hline Effect & Estimate & Standard error & Standard estimate & $95 \% \mathrm{Cl}$ & $P$ value \\
\hline \multicolumn{6}{|l|}{ Model 1} \\
\hline \multicolumn{6}{|c|}{ On VAS pain $\left(R^{2}=0.265\right)$} \\
\hline KAM & -2.50 & 1.61 & -0.21 & $-5.66,0.67$ & 0.12 \\
\hline KFM & 2.26 & 0.89 & 0.35 & $0.51,4.01$ & 0.01 \\
\hline Severity & 1.09 & 0.45 & 0.31 & $0.20,1.98$ & 0.02 \\
\hline \multicolumn{6}{|c|}{ On KAM $\left(R^{2}=0.365\right)$} \\
\hline Varus angle & 0.02 & 0.00 & 0.74 & $0.01,0.03$ & $<0.01$ \\
\hline Walking speed & 0.13 & 0.11 & 0.16 & $-0.07,0.34$ & 0.21 \\
\hline Severity & -0.07 & 0.05 & -0.23 & $-0.16,0.02$ & 0.12 \\
\hline \multicolumn{6}{|c|}{ On KFM $\left(R^{2}=0.192\right)$} \\
\hline Walking Speed & 0.29 & 0.20 & 0.19 & $-0.10,0.68$ & 0.15 \\
\hline KAM & -0.71 & 0.24 & -0.40 & $-1.17,-0.25$ & $<0.01$ \\
\hline \multicolumn{6}{|l|}{ Model 2} \\
\hline \multicolumn{6}{|c|}{ On KOOS pain $\left(R^{2}=0.036\right)$} \\
\hline KAM & -18.06 & 15.04 & -0.19 & $-47.53,11.41$ & 0.23 \\
\hline KFM & -7.76 & 8.34 & -0.15 & $-24.11,8.59$ & 0.35 \\
\hline Severity & 1.04 & 4.25 & 0.04 & $-7.29,9.38$ & 0.81 \\
\hline \multicolumn{6}{|c|}{ On KAM $\left(R^{2}=0.358\right)$} \\
\hline Varus angle & 0.02 & 0.00 & 0.74 & $0.01,0.03$ & 0.02 \\
\hline Walking speed & 0.13 & 0.11 & 0.16 & $-0.08,0.34$ & 0.21 \\
\hline Severity & -0.07 & 0.05 & -0.23 & $-0.16,0.02$ & 0.15 \\
\hline \multicolumn{6}{|c|}{ On KFM $\left(R^{2}=0.183\right)$} \\
\hline Walking Speed & 0.28 & 0.20 & 0.19 & $-0.11,0.67$ & 0.16 \\
\hline KAM & -0.69 & 0.24 & -0.39 & $-1.16,-0.23$ & $<0.01$ \\
\hline
\end{tabular}


Table 4 Effects of external knee adduction moments on intensity of pain measured by VAS and KOOS

\begin{tabular}{|c|c|c|c|c|c|}
\hline & Estimate & Standard error & Standard estimate & $95 \% \mathrm{Cl}$ & $P$ value \\
\hline \multicolumn{6}{|l|}{ VAS } \\
\hline Direct & -2.50 & 1.61 & -0.21 & $-5.66,0.67$ & 0.12 \\
\hline Indirect & -1.61 & 0.83 & -0.14 & $-3.23,0.02$ & 0.05 \\
\hline Total & -4.10 & 0.16 & -0.35 & $-7.21,-1.00$ & 0.01 \\
\hline \multicolumn{6}{|l|}{ KOOS } \\
\hline Direct & -18.06 & 15.04 & -0.19 & $-47.53,11.41$ & 0.23 \\
\hline Indirect & 5.38 & 6.07 & 0.08 & $-6.52,17.27$ & 0.38 \\
\hline Total & -12.69 & 14.09 & -0.13 & $-40.30,14.93$ & 0.37 \\
\hline
\end{tabular}

We used the KAM index as an estimation of the percentage of KAM to the sum of KAM and KFM and found a negative relationship between this index and VAS pain intensity when adjusting for radiographic severity, knee varus angle and walking speed. This concurred with the results from path analysis that participants with lower share of mechanical loading on the frontal plane had less pain in walking. Since this was a cross-sectional observational study, a causal relationship could not be established. There could be other de-loading mechanisms adopted by the participants in response to painful stimuli via re-distribution of load between the frontal and sagittal planes, or higher load sharing on the frontal plane could have happened to minimize the walking pain. However, greater percentage of KAM to total external knee moment was linked with radiographic joint structural degeneration as measured by KL grading in 5 years [23]. Considering this point with the present findings, a higher KAM index would therefore be detrimental to the knee joint in the long run; its apparent association with lower pain was more likely to be associated with some other potential pain avoidance strategies, for instance, knee kinematics asymmetry between two limbs in mild-to-moderate knee OA [35]. However, this study cannot answer this question and further study is warranted to examine the mechanisms of how KAM index would modulate the pain intensity.

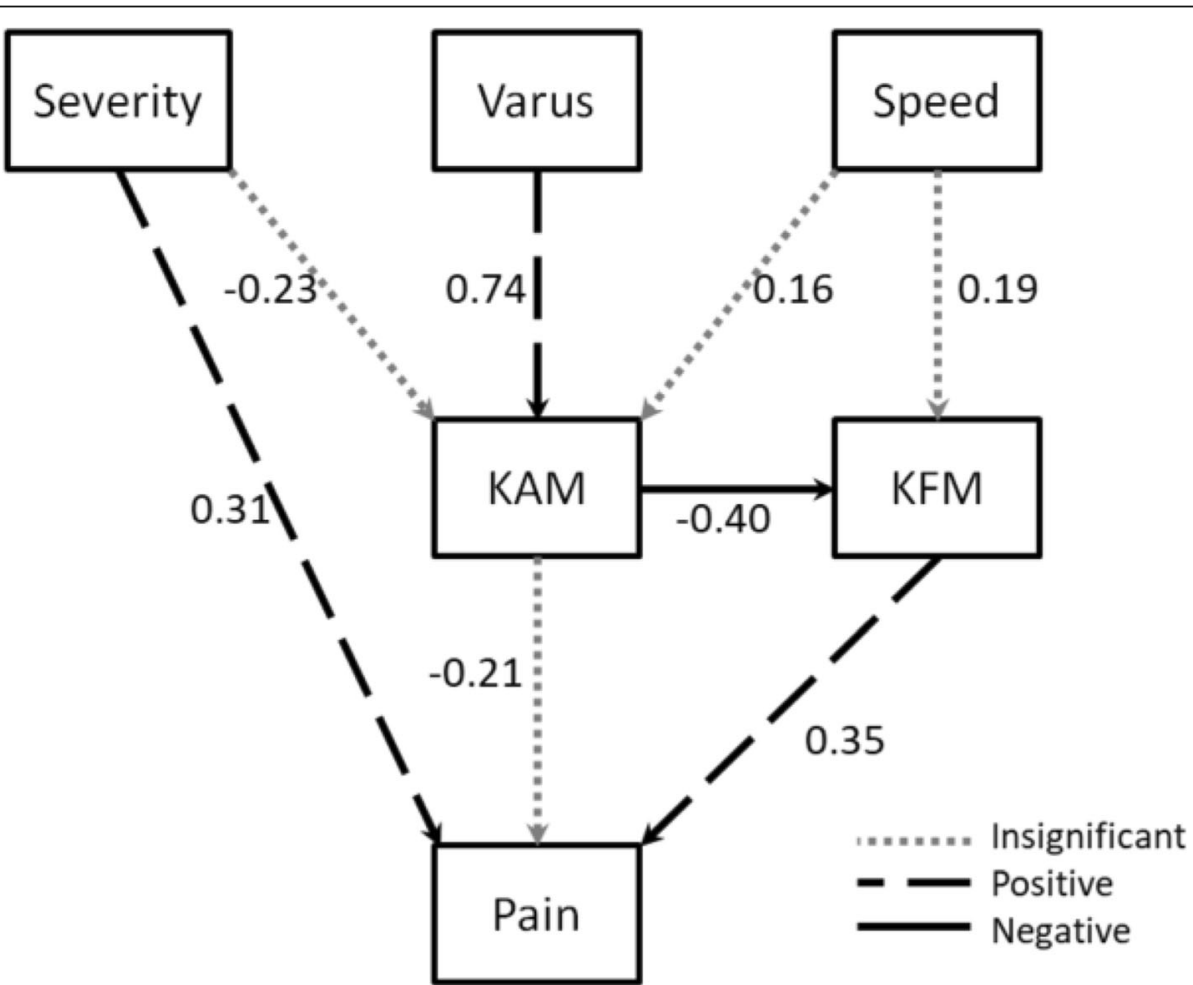

Fig. 1 Diagram of path analysis. (Pain: intensity of pain using visual analogue scale. KAM: external knee adduction moment. KFM: external knee flexion moment. Varus : knee varus angle) 

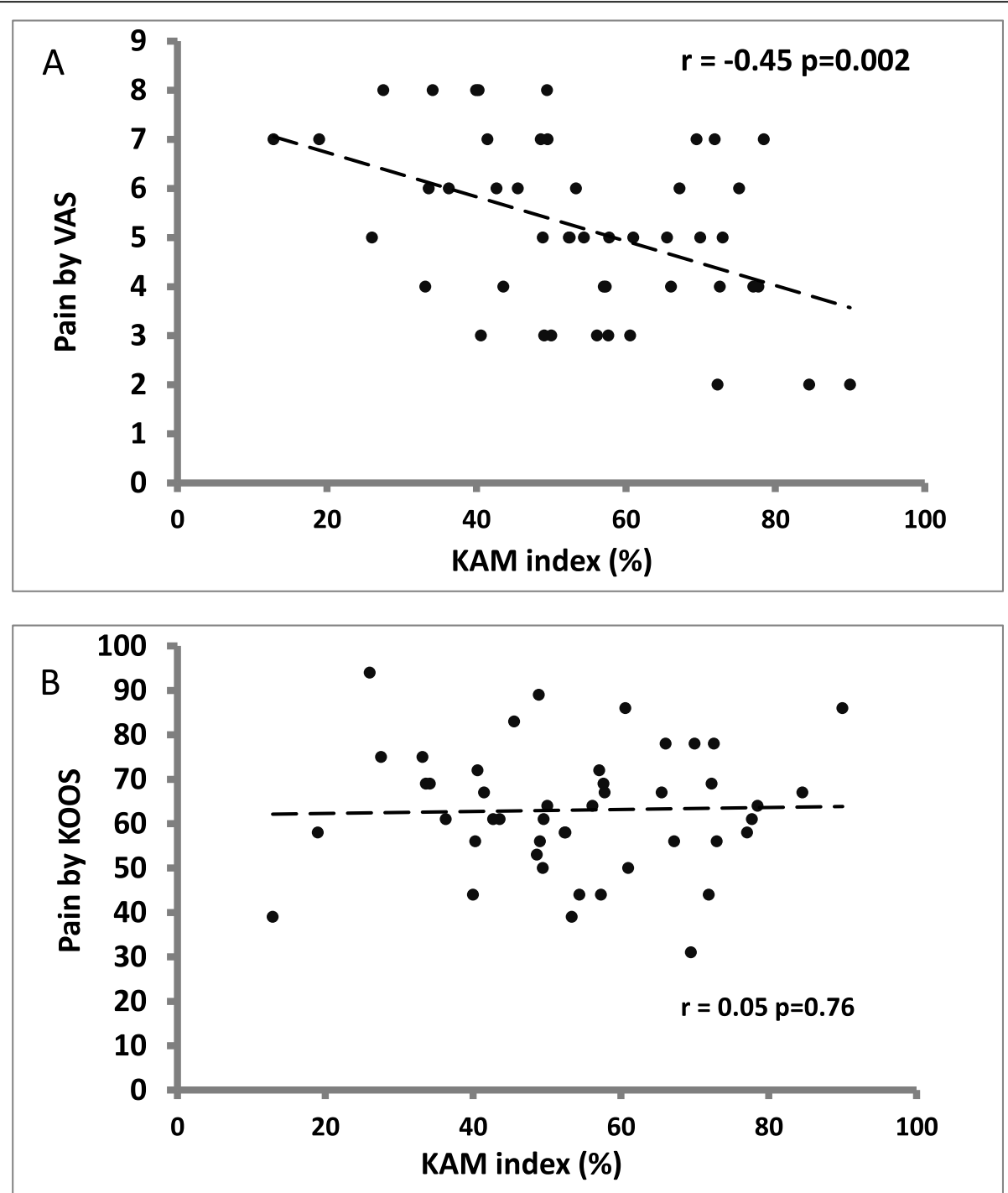

Fig. 2 Scatter plots between pain intensity and KAM index controlling for disease severity, knee varus angle and walking speed. (VAS: visual analogue scale; KOOS: Knee Injury and Osteoarthritis Outcome Score)

The KFM was found to be a mediator for the relationship between KAM and pain intensity in mild-tomoderate knee OA. In view KAM and KFM were antagonistic to one another, the present finding of a positive relationship between pain intensity and KFM echoed with the report of O'Connell et al. [33] that higher KFM was demonstrated in participants with moderate-to-severe pain than those with less pain [33]. Previous study had reported KFM was more sensitive to change in pain intensity than KAM over time in people with medial compartment OA [28]. The KFM was balanced with contraction of the quadriceps muscle which produced an internal knee extension moment [36]. The internal knee extension moment resulted from quadriceps contraction would induce a compressive force across the tibiofemoral joint [37] that might trigger pain.
Interestingly, the KOOS pain intensity and KAM were not likely interrelated either directly or indirectly. Likewise, pain intensity measured with the pain subscale of Western Ontario and McMaster Universities Osteoarthritis Index (WOMAC) was also not associated with the magnitude of KAM in radiographic medial compartment knee OA [38, 39]. A possible explanation might be because VAS was a unidimensional pain measurement tool focused on pain intensity localized at the knee joint whereas both KOOS and WOMAC were multidimensional measuring tools with more emphasis on disease progression and joint function. In fact, the KOOS pain subscale was an extension of WOMAC pain subscale, and they both measured pain intensity with Likert-type scales during several daily activities, including but not limited to level walking [40]. Apart from that, questions 
in KOOS and WOMAC pain subscales were not focused on a specific knee; thereby the pain on both knees for those with bilateral knee OA would influence the outcome. Though it is clarified that area of interest of is the knee in KOOS, low back pain and other musculoskeletal pain could also have an impact on the pain intensity scores when doing these physical activities [41]. Considering these holistic factors, KOOS and WOMAC pain subscales might have weaker relationship with magnitude of joint loading variables than knee pain in walking.

There were some limitations in this study that should be addressed. First, the sample size was considered small especially that of KL grade 3, which had restricted the model building. If the sample size was larger, a more comprehensive model could have been built. Second, the fact that severe knee OA participants were not included in the study has restricted the findings to be only applicable to patients with radiographic knee OA with KL grade less than 4 . Finally, due to the cross-sectional nature of the study, measurements at a time point could not justify if any causal relationship did exist between pain intensity, KAM and other variables. Future interventional study will shed lights on how changes on KAM, KFM and pain intensity are interrelated.

\section{Conclusions}

To conclude, in people with radiographic mild-tomoderate knee OA, KAM had a negative effect on selfperceived intensity of walking-related pain with KFM as its mediator. In these people, greater KAM index was associated with less walking pain. The distribution of knee joint loading from frontal to sagittal planes could be a pain avoidance strategy which has an application value for management of pain in people mild-to-moderate knee OA.

\section{Abbreviations}

KAM: External knee adduction moment; KFM: External knee flexion moment; KL: Kellgren-Lawrence grading scale; KOOS: Knee Injury and Osteoarthritis Outcome Score; OA: Osteoarthritis; VAS: Visual analogy scale:

WOMAC: Western Ontario and McMaster Universities Osteoarthritis Index

\section{Acknowledgements}

The authors would like to thank Mrs. Harriot Ko for her assistance in data collection and Dr. Raymond Chung for his assistance in statistical analysis. The authors also would like to acknowledge Prof. Gabriel Ng for revising and editing the manuscript.

\section{Authors' contributions}

$\mathrm{CH}$ and SNF designed the study. PKC, KYC and CHY provided patient sources. CH and SSY did data collection. CH and SNF did statistical analysis. $\mathrm{CH}$ drafted the initial manuscript. PKC, KYC, CHY, SSY and SNF provided critical revision. All authors gave final approval.

\section{Funding}

None.

\section{Availability of data and materials}

The datasets used and/or analyzed during the current study are available from the corresponding author on reasonable request.

\section{Declarations}

Ethics approval and consent to participate

This study followed the principle of the Declaration of Helsinki and was approved by the Human Subjects Ethics Sub-committee, Department of Rehabilitation Sciences, The Hong Kong Polytechnic University (Ref. HSEA RS20170406003). The procedures were explained to the participants and they all gave their written informed consents prior to commencement of the study

\section{Consent for publication}

Not applicable.

\section{Competing interests}

The authors declare that they have no competing interests.

\section{Author details}

${ }^{1}$ Department of Rehabilitation Sciences, The Hong Kong Polytechnic University, Hung Hom, Hong Kong, China. ${ }^{2}$ Department of Orthopaedics and Traumatology, Queen Mary Hospital, The University of Hong Kong, Hong Kong, China. ${ }^{3}$ Physiotherapy Department, MacLehose Medical Rehabilitation Centre, Hong Kong, China.

Received: 20 April 2021 Accepted: 7 August 2021

Published online: 12 August 2021

\section{References}

1. James SL, Abate D, Abate KH, Abay SM, Abbafati C, Abbasi N, Abbastabar H, Abd-Allah F, Abdela J, Abdelalim A, et al. Global, regional, and national incidence, prevalence, and years lived with disability for 354 diseases and injuries for 195 countries and territories, 1990-2017: a systematic analysis for the Global Burden of Disease Study 2017. Lancet. 2018;392(10159):1789-858.

2. Felson DT: The sources of pain in knee osteoarthritis. Current opinion in rheumatology 2005, 17(5):624-628.

3. Nguyen U-SD, Zhang Y, Zhu Y, Niu J, Zhang B, Felson DT: Increasing prevalence of knee pain and symptomatic knee osteoarthritis: survey and cohort data. Annals of internal medicine 2011, 155(11):725-732.

4. Peat G, McCarney R, Croft P: Knee pain and osteoarthritis in older adults: a review of community burden and current use of primary health care. Annals of the Rheumatic Diseases 2001, 60(2):91.

5. Liu Q, Wang S, Lin J, Zhang Y. The burden for knee osteoarthritis among Chinese elderly: estimates from a nationally representative study. Osteoarthritis and Cartilage. 2018;26(12):1636-42.

6. Stubbs B, Hurley M, Smith T: What are the factors that influence physical activity participation in adults with knee and hip osteoarthritis? A systematic review of physical activity correlates. Clinical Rehabilitation 2015, 29(1):80-94.

7. Alkan BM, Fidan F, Tosun A, Ardıçoğlu Ö: Quality of life and self-reported disability in patients with knee osteoarthritis. Modern Rheumatology 2014, 24(1):166-171

8. Neogi T: The epidemiology and impact of pain in osteoarthritis. Osteoarthritis and Cartilage 2013, 21(9):1145-1153.

9. Hawker GA, Stewart L, French MR, Cibere J, Jordan JM, March L, SuarezAlmazor M, Gooberman-Hill R: Understanding the pain experience in hip and knee osteoarthritis - an OARSI/OMERACT initiative. Osteoarthritis and Cartilage 2008, 16(4):415-422.

10. Felson DT: Osteoarthritis as a disease of mechanics. Osteoarthritis Cartilage 2013, 21(1):10-15

11. Silverwood V, Blagojevic-Bucknall M, Jinks C, Jordan JL, Protheroe J, Jordan KP: Current evidence on risk factors for knee osteoarthritis in older adults: a systematic review and meta-analysis. Osteoarthritis and Cartilage 2015, 23(4): 507-515.

12. Amin S, Luepongsak N, McGibbon CA, LaValley MP, Krebs DE, Felson DT: Knee adduction moment and development of chronic knee pain in elders. Arthritis care \& research 2004, 51(3):371-376.

13. Astephen JL, Stanish WD, Hubley-Kozey CL: Asymptomatic and symptomatic individuals with the same radiographic evidence of knee osteoarthritis walk with different knee moments and muscle activity. Journal of orthopaedic research: official publication of the Orthopaedic Research Society 2017, 35(8):1661-1670. 
14. Hall M, Bennell KL, Wrigley TV, Metcalf BR, Campbell PK, Kasza J, Paterson KL, Hunter DJ, Hinman RS. The knee adduction moment and knee osteoarthritis symptoms: relationships according to radiographic disease severity. Osteoarthritis and Cartilage. 2016;25(1):34-41.

15. Henriksen M, Aaboe J, Bliddal H: The relationship between pain and dynamic knee joint loading in knee osteoarthritis varies with radiographic disease severity. A cross sectional study. Knee 2012, 19(4):392-398.

16. Thorp LE, Sumner DR, Wimmer MA, Block JA: Relationship between pain and medial knee joint loading in mild radiographic knee osteoarthritis. Arthritis and rheumatism 2007, 57(7):1254-1260.

17. Boyer KA, Hafer JF: Gait mechanics contribute to exercise induced pain flares in knee osteoarthritis. BMC Musculoskeletal Disorders 2019, 20(1):107

18. Manal K, Gardinier E, Buchanan TS, Snyder-Mackler L: A more informed evaluation of medial compartment loading: the combined use of the knee adduction and flexor moments. Osteoarthritis and Cartilage 2015, 23(7): 1107-1111.

19. Simic M, Wrigley $T$, Hinman $R$, Hunt M, Bennell $K$ : Altering foot progression angle in people with medial knee osteoarthritis: the effects of varying toe-in and toe-out angles are mediated by pain and malalignment. Osteoarthritis and Cartilage 2013, 21(9):1272-1280.

20. Uhlrich SD, Silder A, Beaupre GS, Shull PB, Delp SL: Subject-specific toe-in or toe-out gait modifications reduce the larger knee adduction moment peak more than a non-personalized approach. Journal of Biomechanics 2018, 66: 103-110.

21. Hurwitz D, Ryals A, Case J, Block J, Andriacchi T: The knee adduction moment during gait in subjects with knee osteoarthritis is more closely correlated with static alignment than radiographic disease severity, toe out angle and pain. Journal of orthopaedic research 2002, 20(1):101-107.

22. Robbins SM, Maly MR: The effect of gait speed on the knee adduction moment depends on waveform summary measures. Gait \& posture 2009, 30(4):543-546

23. Asay JL, Erhart-Hledik JC, Andriacchi TP: Changes in the total knee joint moment in patients with medial compartment knee osteoarthritis over 5 years. Journal of Orthopaedic Research 2018, 36(9):2373-2379.

24. Richards RE, Andersen MS, Harlaar J, van den Noort JC: Relationship between knee joint contact forces and external knee joint moments in patients with medial knee osteoarthritis: effects of gait modifications. Osteoarthritis and Cartilage 2018, 26(9):1203-1214.

25. Chang MJ, Jeong HJ, Kang S-B, Chang CB, Yoon C, Shin JY: Relationship Between Coronal Alignment and Rotational Profile of Lower Extremity in Patients With Knee Osteoarthritis. The Journal of Arthroplasty 2018, 33(12): 3773-3777.

26. Collins N, Prinsen C, Christensen R, Bartels E, Terwee C, Roos E: Knee Injury and Osteoarthritis Outcome Score (KOOS): systematic review and metaanalysis of measurement properties. Osteoarthritis and Cartilage 2016, 24(8): 1317-1329.

27. Creaby M, Wang Y, Bennell K, Hinman R, Metcalf B, Bowles K-A, Cicuttini F: Dynamic knee loading is related to cartilage defects and tibial plateau bone area in medial knee osteoarthritis. Osteoarthritis and Cartilage 2010, 18(11): 1380-1385.

28. Asay JL, Boyer KA, Andriacchi TP: Repeatability of gait analysis for measuring knee osteoarthritis pain in patients with severe chronic pain. Journal of Orthopaedic Research 2013, 31(7):1007-1012.

29. Illés T, Somoskeöy S: The EOS ${ }^{\mathrm{TM}}$ imaging system and its uses in daily orthopaedic practice. International Orthopaedics 2012, 36(7):1325-1331.

30. Klem L: Path analysis. In: Reading and understanding multivariate statistics. edn. Washington, DC, US: American Psychological Association; 1995: 65-97.

31. Schermelleh-Engel $K$, Moosbrugger $H$, Müller $H$. Evaluating the fit of structural equation models: Tests of significance and descriptive goodnessof-fit measures. Methods of psychological research online. 2003;8(2):23-74.

32. Bentler PM, Bonett DG: Significance tests and goodness of fit in the analysis of covariance structures. Psychological Bulletin 1980, 88(3):588-606.

33. O'Connell M, Farrokhi S, Fitzgerald GK: The role of knee joint moments and knee impairments on self-reported knee pain during gait in patients with knee osteoarthritis. Clinical Biomechanics 2016, 31:40-46.

34. Henriksen M, Graven-Nielsen T, Aaboe J, Andriacchi TP, Bliddal H: Gait changes in patients with knee osteoarthritis are replicated by experimental knee pain. Arthritis Care \& Research 2010, 62(4):501-509.

35. Mills K, Hettinga BA, Pohl MB, Ferber R: Between-Limb Kinematic Asymmetry During Gait in Unilateral and Bilateral Mild to Moderate Knee
Osteoarthritis. Archives of Physical Medicine and Rehabilitation 2013, 94(11): 2241-2247.

36. Creaby M: It's not all about the knee adduction moment: the role of the knee flexion moment in medial knee joint loading. Osteoarthritis and Cartilage 2015, 23(7):1038-1040.

37. Walter JP, D'Lima DD, Colwell CW, Fregly BJ: Decreased knee adduction moment does not guarantee decreased medial contact force during gait. Journal of Orthopaedic Research 2010, 28(10):1348-1354.

38. Maly MR, Costigan PA, Olney SJ: Mechanical factors relate to pain in knee osteoarthritis. Clinical Biomechanics 2008, 23(6):796-805.

39. Astephen Wilson JL, Deluzio KJ, Dunbar MJ, Caldwell GE, Hubley-Kozey CL: The association between knee joint biomechanics and neuromuscular control and moderate knee osteoarthritis radiographic and pain severity. Osteoarthritis and Cartilage 2011, 19(2):186-193.

40. Bellamy N, Buchanan WW, Goldsmith CH, Campbell J, Stitt LW: Validation study of WOMAC: A health status instrument for measuring clinically important patient relevant outcomes to antirheumatic drug therapy in patients with osteoarthritis of the hip or knee. Journal of Rheumatology 1988, 15(12):1833-1840.

41. Suri P, Morgenroth DC, Kwoh CK, Bean JF, Kalichman L, Hunter DJ: Low back pain and other musculoskeletal pain comorbidities in individuals with symptomatic osteoarthritis of the knee: Data from the osteoarthritis initiative. Arthritis Care \& Research 2010, 62(12):1715-1723.

\section{Publisher's Note}

Springer Nature remains neutral with regard to jurisdictional claims in published maps and institutional affiliations.
Ready to submit your research? Choose BMC and benefit from:

- fast, convenient online submission

- thorough peer review by experienced researchers in your field

- rapid publication on acceptance

- support for research data, including large and complex data types

- gold Open Access which fosters wider collaboration and increased citations

- maximum visibility for your research: over $100 \mathrm{M}$ website views per year

At $\mathrm{BMC}$, research is always in progress.

Learn more biomedcentral.com/submissions 